\title{
EXPLORING STRATEGIES FOR CLOSED-LOOP CAVITY FLOW CONTROL
}

\author{
M. Samimy, M. Debiasi, E. Caraballo, J. Malone, J. Little \\ Gas Dynamics and Turbulence Laboratory \\ Department of Mechanical Engineering \\ The Ohio State University \\ 206 West $18^{\text {th }}$ Avenue \\ Columbus, Ohio 43210 \\ Samimy.1@OSU.edu
}

\author{
H. Özbay, M. Ö. Efe, P. Yan, X. Yuan \\ Department of Electrical Engineering \\ The Ohio State University \\ J. DeBonis \\ NASA Glenn Research Center \\ Nozzle Branch
J. H. Myatt and R. C. Camphouse
Air Force Research Laboratory
Air Vehicles Directorate

\begin{abstract}
One of the current three main thrust areas of the Collaborative Center of Control Science (CCCS) at The Ohio State University is feedback control of aerodynamic flows. Synergistic capabilities of the flow control team include all of the required multidisciplinary areas of flow simulations, low-dimensional and reduced-order modeling, controller design, and experimental integration and implementation of the components along with actuators and sensors. The initial application chosen for study is closed-loop control of shallow subsonic cavity flows. We have made significant progress in the development of various components necessary for reduced-order model based control strategy, which will be presented and discussed in this paper. Stochastic estimation was used to show that surface pressure measurements along with the reduced-order model based on flow-field variables can be used for closed-loop control. Linear controllers such as $\mathrm{H}_{\infty}$, Smith predictor, and PID were implemented experimentally with various degrees of success. The results showed limitations of linear controllers for cavity flow with inherent nonlinear dynamics. Detailed experimental work further explored the physics and showed the highly non-linear nature of the cavity flow and the effects of forcing on the flow structure.

\section{Introduction}

The flow control team at the Collaborative Center of Control Science at The Ohio State University is developing tools for the systematic integration of feedback control with active flow control. Feedback control is typically used to stabilize systems or to achieve a desired system output. Possible benefits of the integration of feedback control with active flow control include separation control and virtual aerodynamic shaping. The application chosen for initial study is the control of cavity flow resonance (Samimy et al. 2003a, 2003b) using a synthetic jet type of actuation near the top of the upstream wall of the cavity. The problem is relevant to the Air Force because of structural fatigue in weapons bays caused by cavity

resonance.

Several methods can be used to develop feedback control laws for flow control. For instance, successful approaches to date make use of experimental data for the development of models of the fluid/actuator interactions (e.g. Traub et al. 2003, Patel et al. 2003). The advantage of this method is that the control law can sometimes be developed with a small amount of experimental data. The weakness of this and other approaches for the design of flow-control laws is that these laws are developed either on an ad-hoc basis or for a model that may not sufficiently capture the dynamics of the fluid/actuator interaction over a wide range of operating conditions and desired outputs. In the case of cavity resonance, a more flexible approach of system identification has been used to develop loworder models (Cattafesta et al. 2003, Rowley et al.
\end{abstract}

This paper is declared a work of the U.S. Government and is not subject to copyright protection in the United States. 
2003). Cattafesta et al. (1997) demonstrated the utility of integrating feedback control by demonstrating that closed-loop control of cavity tones requires an order of magnitude less power than open-loop control.

The current effort is focused on a further step in the design of more general and robust control laws by introducing a systematic method for order reduction of models based on the Navier-Stokes equations and for the derivation of control laws based on these models. These simplified models must capture all the relevant dynamics and relationships between the various system components and enable the quantification of the control objective in terms of the system states. To this end, it is usually necessary to consider states that represent additional relationships between the inputs and outputs or between other states. Order reduction techniques are employed to reduce the dimension of the system described by the Navier-Stokes equations, a system of nonlinear partial differential equations, to a system modeled by a much smaller set of ordinary differential equations. The reduced-order model is then used to design feedback control laws. This approach has many advantages, foremost of which is the reduction of an infinite-dimensional system to a system consisting of only a few states.

In our effort, work continues to complete one full cycle composed of obtaining the appropriate simulation data capturing the rich dynamics of the flow field, deriving a reduced-order model with well-defined input/output relationships, designing a corresponding control law, and the experimental validation of the overall system. Quasi-three-dimensional simulations are required to correctly model the dynamics of the cavity, and several cases are underway. In addition, two-dimensional simulation data are used to develop the order-reduction method based on Proper Orthogonal Decomposition. An experimental facility has been constructed and tested for validation of the feedback control law, to characterize the actuator, and to provide data for physical understanding of cavity flows. In addition, several ad-hoc control law designs have been implemented to demonstrate the utility of the experimental facility and provide useful data for studying the control authority of the actuator over a range of Mach numbers.

A brief account of numerical simulation activities will be given in Section 2 followed by a description of the reduced-order modeling effort in Section 3. Control law design and experimental implementation of the controllers as well as some results on flow physics will be presented and discussed in Sections 4 and 5, respectively. Section 6 will provide a summary of activities and findings.

\section{Numerical Simulation}

In order to develop reduced-order models for the flow, detailed flow field data are required. Ideally the data should provide a time history of the flow field in and around the cavity for numerous cases with and without forcing. Computational fluid dynamics (CFD) is ideally suited for this task. Time accurate CFD simulations are being performed using an explicit fourth-order accurate large-eddy simulation method (DeBonis \& Scott 2002a, 2002b). The solution is advanced in time using a low-dispersion Runge-Kutta method. Spatial discretization is done using central differencing and the solution is filtered for stability using the method of Kennedy \& Carpenter (1997). The current simulations use Williamson's three-stage Runge-Kutta scheme (1980) combined with fourthorder differencing and a sixth-order filter. The turbulent motion in the flow field is simulated with a hybrid approach. The boundary layer upstream of the cavity is modeled using a Reynolds Averaged NavierStokes (RANS) method to match the experimentally obtained boundary-layer thickness; the Baldwin-Lomax (1978) mixing-length model is used here. In the cavity, the large-scale structures are computed directly and the small-scale structures are replaced with dissipation.

Actuation is incorporated through a boundary condition on the upstream wall of the cavity, right at the cavity leading edge. Experimental measurements of the actuator's output, both with and without external flow, were used to characterize its effect (Debiasi and Samimy 2003). A simple sinusoidal variation in velocity was found to be a good representation. Density is extrapolated from the domain and total enthalpy is conserved.

Both two- and three-dimensional simulations are being used. It is recognized that any direct simulation of turbulence cannot be done properly with 2-D simulations. Using this 2-D approximation, the vortical structures are unnaturally constrained and cannot stretch or tilt. However, these simulations are valid solutions to the 2-D Navier-Stokes equations and can be used to develop the reduced-order modeling techniques that will be applied to the more accurate 3-D solutions. Results of the two-dimensional simulations with and without forcing were previously reported (Samimy et al. 2003b), and the modeling efforts using these solutions are reported in the next section. Threedimensional solutions that will more accurately portray the flow physics are currently underway.

\section{Reduced-Order Modeling}

To develop closed-loop control laws, a simplified model of the flow must be derived. The Navier-Stokes equations governing the flow are a set of highly non-linear partial differential equations not 
suitable for such purpose. To this aim we adopted the Proper Orthogonal Decomposition (POD) method to derive low dimensional models by using numerically simulated data (Caraballo et al. 2003 and Samimy et al. 2003b). We utilized the snapshot method of Sirovich (1987) to obtain the eigenfunction or POD basis, $\varphi^{n}(\boldsymbol{x})$, of the cavity flow from which the flow field instantaneous realizations $\boldsymbol{q}(\boldsymbol{x}, t)$ can be reconstructed as:

$$
\boldsymbol{q}(\boldsymbol{x}, t)=\sum_{n=0}^{N_{P O D}} a^{n}(t) \boldsymbol{\varphi}^{n}(\boldsymbol{x})
$$

where $N_{P O D}$ is the number of POD modes used. If the instantaneous realizations $\boldsymbol{q}(\boldsymbol{x}, t)$ are available through numerical simulations or experimental measurements, the time coefficients, $a^{n}(t)$, can be obtained as:

$$
a^{n}(t)=\int_{D} \boldsymbol{q}(\boldsymbol{x}, t) \bullet \varphi^{n^{*}}(\boldsymbol{x}) d \boldsymbol{x} .
$$

by projecting realizations onto the empirical eigenfunctions.

In developing a reduced-order model, we are interested in estimating the flow evolution from a given state. We therefore use the Galerkin projection method to derive a reduced system of ODEs from which the time coefficients $a^{n}(t)$ in Eqn. 3.1 and hence the evolution in time of the flow from an initial state can be estimated. The governing flow equations, in this case the compressible form of the Navier-Stokes equations, are thus projected onto the POD basis and the system of ODEs so obtained is then truncated at the number of desired modes.

While the full Navier-Stokes equations are solved in the numerical simulation discussed in Section 2, a simplified set of compressible, isentropic form (Rowley et al. 2001) is used to develop reduced-order models. Using the vector-valued norm approach, the equations for the conservation of mass and momentum can be written as:

$$
\begin{aligned}
& \frac{D c}{D t}+\frac{\gamma-1}{2} c \nabla \cdot \boldsymbol{u}=0 \\
& \frac{D \boldsymbol{u}}{D t}+\frac{2}{\gamma-1} c \nabla c=v \nabla^{2} \boldsymbol{u}
\end{aligned}
$$

where $c$ is the speed of sound, $v$ is the viscosity and $\gamma$ is the ratio of specific heats.

As discussed in Samimy et al. (2003b), our approach is to derive a reduced-order model for the system where the control input appears explicitly. The details of this derivation can be found in (Efe et al. 2003a, 2003b). After performing the Galerkin projection with the control or forcing input applied at a specific location of the flow domain, the following system of ODEs is obtained:

$$
\begin{aligned}
& \dot{a}^{k}(t)=b^{k}+\sum_{j=1}^{n}\left(c^{j k} a^{j}\right)+\sum_{j=1}^{n} \sum_{m=1}^{n}\left(d^{j m k} a^{j} a^{m}\right) \\
& +\left\langle e^{k}, \Gamma\right\rangle_{s 1}+\sum_{j=1}^{n}\left\langle f^{j k}, \Gamma\right\rangle_{s 1} a^{j}
\end{aligned}
$$

The constant coefficients $b, c, d$, $e$ and $f$ are obtained from the Galerkin projection while

$$
\Gamma=\left[\begin{array}{l}
\Gamma_{u}(t) \\
\Gamma_{v}(t) \\
\Gamma_{c}(t)
\end{array}\right]
$$

is the control input applied at the forcing location. Defining the velocity of the forcing flow in the numerical simulation as $V=\mathcal{A} \sin (2 \pi f t)$, with $f$ and $\mathcal{A}$ respectively the forcing frequency and amplitude, and computing the corresponding temperature $T$ for this flow (assumed isentropic) from the stagnation temperature, the components $\Gamma_{u}(t)$ and $\Gamma_{v}(t)$ are obtained as:

$$
\begin{aligned}
& \Gamma_{u}=V \cos (\alpha) \\
& \Gamma_{v}= \begin{cases}V \sin (\alpha), & \mathrm{V}>0 \\
0, & \mathrm{~V} \leq 0\end{cases}
\end{aligned}
$$

with $\alpha=30^{\circ}$, while $\Gamma_{c}(t)$ is the nondimensional local speed of sound $c=\frac{\sqrt{\gamma R T}}{c i} \approx 1$.

To design the feedback controller, the systems of equations 3.4 can be recast in control theory notation as

$$
\dot{a}(t)=A(a)+B(a, \Gamma)
$$

where the matrices $A$ and $B$ have the following form (see Efe et al. 2003b):

$$
\begin{gathered}
A(a)=\widetilde{F}+\widetilde{G} a+a^{\mathrm{T}} \widetilde{\mathrm{H}}_{k} a \\
B(a, \Gamma)=\widetilde{P} \Gamma+\widetilde{Q}_{j} \Gamma a
\end{gathered}
$$

and $\widetilde{F}, \widetilde{G}, \widetilde{P}, \widetilde{H}_{k}$ and $\widetilde{Q}_{j}$ are in the form:

$$
\begin{aligned}
& a=\left[\begin{array}{c}
a^{1} \\
a^{2} \\
: \\
a^{n}
\end{array}\right] \quad \widetilde{F}=\left[\begin{array}{c}
b^{1} \\
b^{2} \\
: \\
b^{n}
\end{array}\right] \quad \widetilde{G}=\left[\begin{array}{cccc}
d^{11} & d^{12} & . . & d^{1 n} \\
d^{21} & d^{22} & & d^{2 n} \\
: & & & \\
d^{n 1} & & & d^{n n}
\end{array}\right] \\
& \widetilde{H}_{k}=\left[\begin{array}{cccc}
g^{11 k} & g^{12 k} & . . & g^{1 n k} \\
g^{21 k} & g^{22 k} & & g^{2 n k} \\
\vdots & & & \\
g^{n 1 k} & & & g^{n n k}
\end{array}\right] \\
& \widetilde{P}=\left[\begin{array}{ccc}
e^{11} & e^{12} & e^{13} \\
e^{21} & & \\
\vdots & & \\
e^{n 1} & . . & e^{n 3}
\end{array}\right] \tilde{Q}(a)=\left[\begin{array}{c}
a^{\mathrm{T}} Q_{1} \\
\vdots \\
a^{\mathrm{T}} Q_{n}
\end{array}\right]
\end{aligned}
$$


where $\mathrm{k}$ is the mode number and $\mathrm{i}, \mathrm{j}$ and $\mathrm{k}=1,2, \ldots \mathrm{n}$. As was discussed in Samimy et al. (2003b), we encountered some convergence problems in the solution of the system of ODEs obtained from the Galerkin projection for some of the flow cases. In addition to the least square method used in our previous work, an alternative method, based on the weak formulation of the governing equation, is being developed to understand and improve the solution. A brief discussion of this formulation and of how it may address potential issues relevant to our problem is given in the following.

There are two difficulties associated with the reduced-order model described above. One is that this model requires second order spatial derivatives of the POD basis functions. Since higher order POD modes can be very oscillatory, the accurate numerical approximation of their second order derivatives can be quite difficult. As a result, errors may be introduced into the model if high order modes are included. The second difficulty is associated with the control location in the cavity geometry. In our case, the control entry is located strictly on the boundary of the cavity. The method used to extract control inputs in the reduced order model above relies on a repartitioning of the geometry, namely the underlying fact that

$$
\int_{\Omega} g d x=\int_{\Omega_{1}} g d x+\int_{\Omega \backslash \Omega_{1}} g d x, \text { for } g \in L^{2}(\Omega),
$$

where $\Omega_{1}$ is the portion of the domain over which the control is located, i.e., $g(t, \mathbf{x})=u(t)$ for $\mathbf{x} \in \Omega_{1}$ and $u(t)$ is the control. This repartitioning is suited well for the case of distributed control, i.e., the control over a positive area or volume. However, in the case of boundary control, the efficacy of this partition is less apparent as the control domain $\Omega_{1}$ has zero measure and, as a result,

$$
\int_{\Omega_{1}} g d x=0 .
$$

Therefore, it is somewhat unclear how well the control input of the reduced order model can be extracted via a repartition in this case. Extension of the control from the boundary to a distributed region of positive measure may change the problem significantly.

The weak formulation of the reduced order model provides a natural means to overcome the difficulties described above as it reduces the derivative requirements and provides a way to extract control inputs when the control is located on the boundary. The basic idea behind the weak formulation is to integrate the reduced-order model by parts. The control input can be made explicit in the resulting boundary terms while only first-order spatial derivatives of the POD modes are required by the weak form. To determine the feasibility of this approach, we applied it to a simple 1-D heat equation, an initial boundary value problem. Control inputs entered the problem in the form of Dirichlet boundary conditions. We developed a POD model for the problem and constructed its weak form. We then considered a linear quadratic regulator control formulation for the weak POD model. We specified that the solution track a given reference signal. The feedback control obtained through this formulation was very effective when applied to the reduced-order model and tracking was easily achieved. To determine the effectiveness of the reduced-order control in the "real" full order problem, we implemented the reduced-order feedback control in the full-order simulation. The reduced-order control was effective there as well. The full-order simulation with the reduced-order feedback control tracked the reference signal very well. This was the case even when the reduced-order control was determined from a weak POD model consisting of only three modes. Specific details about this problem and its control are the subject of a forthcoming paper. We are now extending these ideas to the cavity flow problem.

\subsection{Feedback Control with Surface Pressure as the System Output}

In practical applications, a surface variable, such as surface pressure, will be measured. The reduced-order model, on which the controller law is based, must use this measurement data to find the state of the flow and then to determine the actuator action. Therefore, the reduced-order model must be based on the surface pressure, or, on the flow field variables (e.g. the flow velocity). In the latter case, a relation between the flow variables and the surface pressure must be established first. To obtain a relation between the surface pressure and the flow field velocity within the cavity flow, we used the stochastic estimation method with the numerically simulated data. The stochastic estimation method was developed by Adrian (1977) to capture coherent structures in turbulent flows, and it has been used by many researchers in various flows (e.g. Adrian and Moin 1988, Cole et al. 1991, Cole and Glauser 1998). Several researchers used it as a complementary technique to obtain the POD time coefficients; for example, in subsonic jets (e.g. Picard and Delville, 2000) and in cavity flows (Murray and Ukeiley, 2003). The procedure followed here is similar to that of Murray and Ukeiley (2003).

The estimation is obtained using known surface pressure and flow field variables (velocities) from simulation results. The goal is to obtain a set of coefficients that will correlate these two sets of variables and will allow the estimation of the flow field variables based on the time dependent measured surface 
pressure. The stochastic estimation provides the following relation:

$$
\begin{aligned}
& \widetilde{q}_{i j}(t)=C_{i j k} P_{k}(t)+D_{i j l m} P_{l}(t) P_{m}(t) \\
& +E_{i j p r s} P_{p}(t) P_{r}(t) P_{s}(t)+\cdots
\end{aligned}
$$

where $\widetilde{q}_{i j}$ is an estimated flow variable vector (velocity), $P_{k}$ is the instantaneous pressure at the surface of the cavity. The subscript indices $i$ and $j$ represent the different spatial locations, and $k, l, m, p, r$, $s, \ldots$ correspond to the selected surface pressure locations (from 1 to the total number of pressure locations). The coefficient matrices $(C, D, E, \ldots)$ for the stochastic estimation are obtained from the correlation of the instantaneous flow field with the surface pressure, with zero lag, and by minimizing the mean square error between the real value $q_{i j}$ and the estimated one $\widetilde{q}_{i j}$

$$
e_{i j}=\left\langle\left[\widetilde{q}_{i j}-q_{i j}\right]^{2}\right\rangle
$$

In Eqn. 3.12 we retained both the linear and the quadratic terms since the latter significantly improved the results. In our test we considered only four surface pressure measurement locations, three within the cavity floor and one on the wall of the incoming flow, without optimizing their location or number. The equations correlating the two velocity components and the speed of sound in the cavity to the four measured surface pressures at a given time, $\mathrm{t}_{\mathrm{r}}$ are

$$
\begin{aligned}
& \tilde{u}_{i j}\left(t_{r}\right)=C_{u i j k} P_{k}\left(t_{r}\right)+D_{u i j l m} P_{l}\left(t_{r}\right) P_{m}\left(t_{r}\right) \\
& \widetilde{v}_{i j}\left(t_{r}\right)=C_{v i j k} P_{k}\left(t_{r}\right)+D_{v i j l m} P_{l}\left(t_{r}\right) P_{m}\left(t_{r}\right) \\
& \widetilde{c}_{i j}\left(t_{r}\right)=C_{c i j k} P_{k}\left(t_{r}\right)+D_{c i j l m} P_{l}\left(t_{r}\right) P_{m}\left(t_{r}\right)
\end{aligned}
$$

It should be noted that in Eqn. 3.14 the (estimation) coefficient matrices differ for each of the flow variables used in the reduced-order model. This set of equation gives the instantaneous state of the flow over the entire cavity, based on the pressure at the four sensing locations.

We tested the estimation procedure above using the numerically simulated pressure and velocity data. Taking the pressure at any given time at the four locations, the velocity field at every location in the field was estimated using Eqn. 3.14. Then the time coefficients corresponding to this state were obtained by projecting the estimated velocities onto the POD basis for each variable using Eqn. 3.2. These estimated time coefficients can then be used as the initial condition to solve the system of ODE's to obtain the evolution of the time coefficients (Eqn. 3.4). Finally, the advanced state of the flow can be predicted using the resultant time coefficients in the POD approximation, with Eqn. 3.1. As a result, it would be possible to compare the state obtained for the flow with the desired state and hence to design a control algorithm that modifies the flow accordingly. A simplification in the comparison would be obtained if a value for the pressure at any of the sensing locations used for control purposes could be obtained from the state predicted for the flow. For this purpose an additional relation between the pressure $P$ at each sensing location and the estimated/predicted time coefficient $a^{n}$ corresponding to the $\mathrm{n}^{\text {th }}$ POD mode can be obtained by using stochastic estimation. In this case linear terms are sufficient for good results, and the estimated pressure can be obtained as

$$
\widetilde{P}(t)=M_{n} a^{n}(t)
$$

where $M_{n}$ is the estimation coefficient matrix between the pressure and the $\mathrm{n}^{\text {th }}$ time coefficient. The results obtained for the estimated pressure show that between 3 and 5 time coefficients are sufficient to obtain a good estimate of the pressure.

In summary, after all the coefficient matrices have been calculated from numerical simulation data, the procedure to obtain the flow field behavior, velocities and pressure at the measuring location, based on the pressure to control the flow, would be as follows:

1. Measure the pressure at the sensing locations. (We have not yet optimized the number or the locations of the pressure sensors).

2. Estimate the instantaneous velocity field, based on the quadratic stochastic estimation. (Eqn. 3.14)

3. Calculate the instantaneous time coefficient (Eqn. 3.2) corresponding to the estimated velocity field that will be used as the initial condition for the system of ODE's.

4. Solve the system of ODE's (Eqn. 3.4).

5. Reconstruct the flow field using the POD approximation (Eqn. 3.1)

6. Obtain the estimated pressure at one or more of the locations in step 1 using (Eqn. 3.15).

7. Compare the predicted pressure with the desired one to define the appropriate control action.

\subsection{Sample Results}

In Figure 3.1 we present the first time coefficient obtained from the solution of the system of equations 3.4 with the explicit control input using the first five POD modes. It can be seen that, at least for the first 4 cycles, the solution converges very well for the baseline (unforced) case as well as for forcing with velocity amplitude $\mathcal{A}=40 \mathrm{~m} / \mathrm{s}$ at the two frequencies of $f=500$ and $900 \mathrm{~Hz}$. A phase shift can be detected after four cycles which gradually increases over time while the coefficient amplitude remains unchanged. Similar results were observed for higher numbers of modes, but 
as was noticed before (Samimy et al. 2003b), after 12 modes the system diverges rapidly.

Figure 3.2 shows a comparison of the first time coefficient for the baseline case obtained using the first five POD modes in three different ways. The original (thick line) corresponds to the value obtained from the numerical data by projecting the flow field onto the POD basis using Eqn. 3.2. The second (dashed) line was obtained using Equation 3.4 and using the actual velocities of the first snapshot as the initial condition, as in Figure 3.1. Finally, the last (thin) line was obtained by using the estimated velocity field, based on the four pressure measurements for the first snapshot and the quadratic estimation (Eqn. 3.14) to define the initial condition for the system of equation. It can be observed that the results obtained from the estimated state based on the pressure measurements reproduce the behavior of the system based on the velocity information of the first snapshot. Figure 3.3 shows that the same behavior is obtained for the two forced cases.

Figures 3.4 and 3.5 present the results of the estimated pressure at the center of the cavity floor, Eqn. 3.15. These plots were constructed similar to Figures 3.2 and 3.3. As in the case of the first time coefficient, it can be noticed that with the stochastic estimation the pressure signal follows the original value very well for all three cases. These encouraging results indicate that it should be possible to identify the state of the flow from a few pressure measurements and to estimate the pressure at the control location such that a suitable controller could be designed and tested.

\section{Controller Design}

In this section, we briefly summarize the results presented in our companion paper Yan et al. (2004), which deals with real time control of cavity flows. We already saw in Section 3 that the system (cavity flow) is represented by a set of nonlinear partial differential equations, which makes it difficult to exploit the powerful tools of classical control theory directly. For this reason, several approaches have been considered for obtaining a reduced-order dynamical model amenable to control design. While we continue developing the nonlinear reduced-order model described in Section 3, we are also considering other possible representations of cavity flow dynamics, and deriving simple controllers.

A linear model for cavity flow oscillations was proposed by Rowley et al. (2002a, 2002b, 2003). It handles the modeling problem from a decomposed process physics point of view. More explicitly, associated dynamical components (e.g. acoustics feedback, shear layer instability, receptivity, acoustic scattering) are individually modeled. Furthermore, Yuan et al. (2003) have shown that the parameters of such a delay based linear model can be tuned in such a way that its open loop system response to a white noise input fits given experimental data.

For the above-mentioned linear model, we designed simple linear controllers such as PID controller, Smith predictor based controller, and $\mathrm{H}_{\infty}$ controller. Experimental results summarized in the companion paper, Yan et al. (2004), outline two important conclusions: (i) all three linear controllers derived from a linear plant model for a single dominant Rossiter mode are able to suppress the cavity oscillations at this mode, but they shift the oscillations to another Rossiter frequency, which was not present explicitly in the unforced (open loop) case; and (ii) adding a zero to the simplest of these controllers, proportional controller, avoids this problem, provided that the location of the zero matches the newly excited Rossiter mode mentioned above.

From these observations, it is obvious that the linear models are not "rich enough" to capture the dynamical system behavior when the feedback loop is closed. Hence, we turn our attention to nonlinear models, such as the one derived in Section 3. Now our immediate goal is to analyze this nonlinear input-stateoutput model, and develop new control design techniques for it. Recall that this nonlinear system is obtained from Proper Orthogonal Decomposition, with a dense grid and sufficient number of snapshots from the process. In this case, modeling errors are relatively small, if sufficiently large number of modes is used. Unfortunately, a POD model cannot synthesize a behavior whose signature is absent in the snapshots. This results in a locality problem, which can be solved by interpolating different POD models that are valid for different operating conditions. One of our plans is to use fuzzy decision mechanisms to interpolate different POD-based models. This approach has been demonstrated successfully on a system represented by the 1D Burgers equations (Efe et al. 2004a). Control of interpolated POD-based nonlinear models is in our list of long-term goals.

Another approach to modeling of aerodynamic flows is the use of neural networks, which are inherently nonlinear. Given a set of input-output data (e.g. excitation voltage applied to the actuator, and pressure readings at several points on the cavity floor/wall) we can develop a neuroidentifier that imitates the input-output behavior of the actual system. This neural structure may have arbitrary number of inputs (down to a finite delay depth in discrete time), arbitrary number of hidden layers, and arbitrary number of hidden neurons. There are a set of choices for nonlinear activation functions and the training schemes. The goal is to set and train a network structure, which has the simplest topology with acceptable performance. A neuroidentifier has already been implemented by 
using limited experimental data; preliminary results are presented in Efe et al. (2004b). The design of neurocontroller under the presence of neuroidentifier is also possible under certain conditions; see Narendra and Parthasarathy (1990). We also plan to design such a controller.

\section{Experiments}

In this section, we briefly discuss the experimental activities and present some experimental results obtained up to date at the Gas Dynamics and Turbulence Laboratory (GDTL) at OSU as part of the CCCS flow control activities. The experimental facility used in this study is described in more detail in Debiasi and Samimy (2003). It consists of an optically accessible, blow-down type wind tunnel capable of continuous operation in the subsonic range with the present set up and in the supersonic range by exchanging the nozzle. The air, supplied by two fourstage compressors, is conditioned in a stagnation chamber before entering the square test section (with width $W=$ height $H=50.8 \mathrm{~mm}$ ) through a smoothly contoured converging nozzle, Fig. 5.1. A cavity that spans the entire width of the test section is recessed in the floor with a depth $D=12.7 \mathrm{~mm}$ and length $L=50.8$ $\mathrm{mm}$ for an aspect ratio $L / D=4$.

The cavity shear-layer is gently forced by a $2 \mathrm{D}$ synthetic-jet type actuator with an average actuator/main flow momentum ratio, $\mathrm{C}_{\mu}$, of about $2 \cdot 10^{-4}$ issuing from a high-aspect-ratio converging nozzle embedded in the cavity leading edge and exhausting at an angle of $30^{\circ}$ with respect to the main flow through a $1 \mathrm{~mm}$ slot spanning the entire cavity. Actuation in the frequency range $1-20 \mathrm{kHz}$ is provided by the titanium diaphragm of a Selenium D3300Ti compression driver. Debiasi and Samimy (2003) observed significant variations of the amplitude of the velocity fluctuations at the exit slot with forcing frequency. As it will be shown, the effect of forcing amplitude is relatively minor and affects the control authority only at higher Mach numbers.

Pressure fluctuations were measured by dynamic pressure transducers placed in different locations in the test section. The reference signal was obtained by a Kulite XTL-190-25A transducer flush-mounted in the middle of the cavity floor. A dSpace 1103 controller board connected to Dell Precision Workstation 650 computer was used to acquire this signal at $50 \mathrm{kHz}$ through a 12-bit channel and to manipulate it to produce the desired control signal from a 14-bit output channel. In order to maximize the control board performance, its processor was used exclusively for running the control routines. Simultaneous recording consisting of 262,144 samples each were obtained through a 16-bit resolution acquisition board (National
Instruments PCI-6036E) operating independently in the computer. Each recording was band-pass filtered between 200 and $20,000 \mathrm{~Hz}$ to remove spurious frequency components and acquired at a sampling frequency of $200 \mathrm{kHz}$. By using the Kulite sensitivity of $4 \mathrm{mV} / \mathrm{PSI}$ and accounting for the amplifier gain setting, the voltage values of the timetraces were converted to non-dimensional pressure referenced to the commonly used value of $20 \mu \mathrm{Pa}$. Eight narrowband power spectra were computed using a 32,768-point fast Fourier transform, which provided a spectral resolution of about $6 \mathrm{~Hz}$, converted to Sound Pressure Level (SPL) spectra, and then averaged.

The instantaneous features of the flow in a streamwise plane at the test section centerline were obtained by the scattering of a laser light sheet from a Continuum Nd:YAG pulsed laser operating at a wavelength of $532 \mathrm{~nm}$ and entering the test section from an optical window on the top wall of the tunnel. Smoke entering the cavity from a streamwise slot at the floor of the cavity was used for flow visualizations. This technique was found to be superior to using laser induced fluorescence from trace acetone molecules seeded into the main flow. Images were acquired using a Princeton Instruments (now Roper Scientific) ICCD camera. The camera and the laser were synched to a reference signal for acquisition of phase-locked images. In the case of forced flow the reference signal was the actuation voltage. In the case of unforced flow (baseline flow) the reference signal was the voltage generated by the vibrating actuator diaphragm/voice-coil assembly picking up the cavity flow resonance. This technique produced a smooth signal retaining only the main pressure fluctuations while naturally rejecting other noise components. For every flow condition explored, 8 sets of phase-locked images were obtained, each corresponding to an incremental phase shift of $1 / 8$ of the resonant or forced period. Ensemble average images were obtained from the individual images of each set. When run in sequence these average images produce short duration movies illustrating the evolution of the shear-layer characteristics during a cycle.

\subsection{Actuator Authority, Flow Controllability, and Cavity Non-linear Dynamics}

Debiasi and Samimy (2003) explored the resonant flow characteristics of the experimental apparatus, Fig. 5.2, and introduced a logic-based control process based on the experimental observations. This process looks for the forcing frequencies that could significantly reduce the noise spectral peak and maintains the system in such conditions until changes in flow conditions (e.g. a change in the flow Mach number) and thus spectral peak level triggers a new search. As summarized in Fig. 5.3 , this control technique performed remarkably well as it was able to reduce strong cavity-flow resonant 
peaks in the Mach 0.25-0.5 range explored. From this figure, we first note that the intensity of the actuation voltage (i.e. the effect of the forcing amplitude) is insignificant for flows up to about Mach 0.4. At higher Mach numbers, especially above Mach 0.45 , the original resonant peak is much stronger and only intense actuation at 4 or $5 \mathrm{~V}_{\text {rms }}$ is capable of producing a peak reduction of $10 \mathrm{~dB}$. This could be a result of the substantially increased low-frequency noise floor and spectral peaks at higher Mach number. This issue will be further explored in the future.

In an attempt to better understand the physics of the forcing mechanism exploited by the logic-based controller, we explored in detail its effects by forcing some flows with frequencies in the 1-6 kHz range. The flows selected were chosen to be below Mach 0.4 to rule out the effect of forcing amplitude discussed above. Figure 5.4 summarizes the results obtained by sweeping the forcing frequency with $10 \mathrm{~Hz}$ increments in the Mach 0.3 single-mode resonant flow (which resonates at $2.8 \mathrm{kHz}$, identified by a dot in the figure). For this flow the SPL peak values are significantly reduced by forcing at frequencies in the neighborhood of $3.3,3.5$, and $4.0 \mathrm{kHz}$. It is also noteworthy that these frequencies do not correspond to the values for which the actuator velocity, measured at the exit slot by a hotfilm, is high (see also Debiasi and Samimy, 2003). This confirms the previous observation that below Mach 0.4 only a small excitation is necessary to force the flow in its receptivity region.

A better appreciation of the effect of the forcing frequency can be obtained by arranging and running the SPL spectra results in a movie-like sequence, from which the peak values shown in Fig. 5.4 were obtained. In Fig. 5.5 we present a few samples of such spectra. Figure 5.5 (a) is the unforced (baseline) Mach 0.3 flow, which is dominated by a $132 \mathrm{~dB}$ resonant peak at about $2.8 \mathrm{kHz}$ corresponding to the $3^{\text {rd }}$ Rossiter mode (Debiasi and Samimy 2003). Actuation at $1.0 \mathrm{kHz}$, Fig. 5.5 (b), did not modify the resonant peak, but introduced several other peaks at the forcing frequency, its first harmonic as well as at two frequencies corresponding to a linear combination of the forcing frequency and the third Rossiter mode (their difference and sum). These non-linear dynamics can be observed for other forcing frequencies in Fig. 5.5 (c) and (d) where the resonant peak remains unchanged but other peaks appear that correspond to harmonics of the forcing frequency and/or its sum/difference with the Rossiter frequency. Forcing at the frequency of 1.83 $\mathrm{kHz}$, corresponding to the second Rossiter modes, Fig. 5.5 (e), destroys the natural feedback mechanism associated with the third Rossiter mode and excites the lower second mode and its harmonics. Finally forcing at $3.92 \mathrm{kHz}$, Fig. 5.5 (f), produces a spectrum devoid of any strong tone where the most significant features are a remnant of the original resonant peak and a modest forcing peak. This case corresponds to one of the optimal forcing conditions discussed above and in Debiasi and Samimy (2003). Analogous results, not presented here, were obtained for other flow conditions with single or multi-mode resonance and exhibit similar non-linear coupling effects of the forcing input with the natural Rossiter modes.

\subsection{Flow Visualization Results}

We used the phase-locked, laser-imaging technique outlined earlier to visualize the effect of forcing on the flow structure. Images of this kind are very useful to verify the modal behavior of the cavity flow and to provide data for development of accurate numerical simulations codes. In Fig. 5.6 we present some selected images corresponding to the forcing cases discussed above. The images on the left are instantaneous (with 9 ns exposure time) while the ones on the right are the corresponding phase-averaged images (average of about 40 instantaneous images). In all images the flow is from let to right.

Figure 5.6 (a) and (b) correspond to the baseline (unforced) Mach 0.3 resonant flow. In both images, three coherent shear-layer structures are clearly visible as expected for the $3^{\text {rd }}$ Rossiter mode at which this flow resonates. Figures 5.6 (c) and (d) refer to the same flow excited at $1830 \mathrm{~Hz}$. i.e. at the second Rossiter mode. As a result two large coherent shear-layer structures are clearly visible in the pictures. This visually confirms the comments made with respect to the spectrum of Fig. 5.5 (e), i.e. that forcing at this frequency destroys the natural feedback mechanism for the third Rossiter mode and excites the flow at this lower mode. Figures 5.6 (e) and (f) present the Mach 0.3 flow forced at $3920 \mathrm{~Hz}$, an optimal frequency for reducing the spectral peaks. This frequency, about $4 / 3$ of the original resonant one, is close to the fourth Rossiter mode and as a result four shear-layer structures are clearly visible in the pictures. Inspection of all the images obtained for this forcing frequency at various phases of the cycle do not reveal any indication of mode switching since four structures remain clearly visible at all times.

Figure 5.7 (a) and (b) are respectively the SPL spectrum and the phase-locked average image of the multi-mode resonating Mach 0.32 flow without forcing. Due to the continuous switching between the second and third Rossiter modes (observed in instantaneous images), in Fig. 5.6 (a) no single mode locks-in to dominate the spectrum (Cattafesta et al. 1997, 1998; Williams et al. 2000). The average image obtained by phase-locking at the frequency of 3220 corresponding to one of the small peaks visible in Fig. 5.6 (a) does not reveal repetitive shear-layer structures, a finding consistent with the mode-switching mechanism. 
Comparison of Figs. 5.5 (e) and (f) and Fig. 5.6 (d) seems to rule out that optimal frequency forcing induces in the system a state similar to multimode resonance. Additional insight is therefore necessary to ascertain the nature of peak noise reduction associated with the forcing at some frequencies.

\subsection{Results from the Implementation of PID Controller}

As was briefly discussed in Section 4 and detailed in the companion paper (Yan et al. 2004), experiments were performed to test the effect of PID control on the resonant cavity flow. The control routine was developed as a Simulink code and implemented in the dSpace system. Initially the best control parameters were determined by manually adjusting the gains of the proportional (P), integral (I), and derivative (D) terms. The integral term did not have any significant effect on the system response, most probably due to the actuator dynamics. It was found that the derivative term could be replaced with a first order filter with an adjustable cut-off frequency to have a PD like controller of the form:

$$
C(s)=K_{p}+K_{d} \frac{\tau_{d} s}{\tau_{d} s+1}
$$

With zero time delay $(\mathrm{h}=0)$, its optimal parameters were found to be $K_{p}=8, K_{d}=0.04$, and $\tau_{d}=200$. This controller was successful in eliminating the main frequency of oscillation (third Rossiter mode), but led to strong oscillations in the neighborhood of the second Rossiter mode, an expected result since this linear controller was designed for the baseline case and thus the potential excitement of the other Rossiter modes was not taken into account. By adding a time delay of $\mathrm{h}=260 \mu$ s to the "derivative" term we introduced a 180 degree phase shift for signals operating in the neighborhood of the second Rossiter mode and we effectively placed a "zero" at the corresponding frequency. A schematic diagram of this controller and its effect in reducing the peaks of the Mach 0.3 flow are given in Fig. 5.8. For ease of comparison in this figure the voltages corresponding to the $\mathrm{P}, \mathrm{D}$, and the control terms were processed to yield spectra similar to the SPL ones from pressure transducers located at the actuator exit and in the cavity floor. Combination of the $260 \mu$ s phase shifted $\mathrm{P}$ and $\mathrm{D}$ signals produces the control signal whose spectrum is characterized by frequency cancellation at $1.93 \mathrm{kHz}$ (zero placement in the neighborhood of the second Rossiter mode). Cancellation occurs also at the odd harmonics of this frequency while modest reinforcement is produced at the even harmonics. The SPL spectrum of the corresponding acoustic signal emerging from the actuator exit slot is rather complex and reflects the effect of the actuator transfer function. It should be noted that the highest spectral peak of this signal occurs at a frequency of about $3.80 \mathrm{kHz}$, a value near a range of optimal frequencies for spectral peak reduction. Due to the relatively large value of the time delay $\tau_{d}$, the PD like controller above acted like a P-P controller with individual delay terms that was also implemented and produced analogous results.

Finally, it is interesting to note that the performance of these controllers was also quite satisfactory at Mach numbers with multi-mode resonance or at higher Mach numbers exhibiting stronger, single-mode resonance. For the discussion of the additional linear controllers of the Smith-predictor and $\mathrm{H}_{\infty}$ form, the reader is referred to the companion paper by Yan et al. (2004).

\section{Concluding Remarks}

The main objective of the flow control team at the Collaborative Center of Control Science at OSU is to develop tools and methodologies for feedback control of aerodynamic flows enabling the control of flow over air vehicles. The team, composed of OSU, Air Force Research Laboratory, and NASA researchers, is taking a multidisciplinary approach by assembling people with various skills in relevant fields to tackle from the outset this challenging problem in a coordinated fashion. The initial application chosen for study is control of the large-amplitude pressure fluctuations created in a shallow subsonic cavity flow. The cavity flow has long been an attractive problem for researchers due to the rich nature of its flow physics and its relevance to many practical applications, and it has been explored over several decades.

The coordinated efforts undertaken by our team include:

- numerical simulation of the cavity flow,

- development of low-order model of the flow (currently using data from simulation results, and later on from experimental results as well),

- design of control laws based on the reduced-order as well as other models of the flow,

- an experimental effort to provide further understanding of the physics of the flow, to evaluate the simulation results, and to implement and test the overall control schemes.

Significant progress has been made in all components, which was presented and discussed.

Both 2-D and quasi-3-D simulations of the cavity are being pursued. Two-dimensional simulations provide quick results, but often do not capture the correct physics of the cavity flows. Therefore, 2-D simulations are being used for preliminary modeling work in order to explore various issues associated with reduced-order model development and to build up the 
tools that will be used with the three-dimensional simulation results when they become available. To date, 2-D simulations are complete for two baseline (no actuation) cases with Mach numbers 0.30 and 0.38 , and two forced cases using a synthetic jet type actuation for each of the baseline cases.

It is desirable (perhaps required) from the controller design viewpoint to have separate control input terms in the reduced-order model equation, rather than having an autonomous reduced-order model equation. Mach 0.38 baseline and two forced cases were used to look into this issue. These simulation results along with a stochastic estimation technique were used to investigate the potential use of surface pressure measurements with flow-field based reducedorder model for feedback control purpose. The results are quite encouraging.

While the work on the development of reducedorder-model based controller design is being continued, linear controllers such as $\mathrm{H}_{\infty}$, Smith predictor, and PID were implemented experimentally with various degrees of success. The results showed limitations of linear controllers for cavity flow.

Our earlier experimental work with logic-based controller was continued to explore the physics of the shallow cavity flows. We also added flow visualizations to aid us in this task. The flow forcing results are very interesting and clearly show the non-linear dynamics of the shallow cavity flow. Forcing the flow using a synthetic jet type actuator at the leading edge of the cavity at various frequencies, spanning from close to the first Rossiter mode to sixth Rossiter mode, produces a wide variety of interesting results from total suppression of the single dominant mode (third Rossiter mode for Mach 0.3 case) to adding various other peaks. Interestingly, all the peaks are related to the forcing frequency, Rossiter modes, or a combination of the forcing frequency and the dominant Rossiter mode, a clear indication of non-linear behavior of the cavity flow. Phase-locked planar laser sheet flow visualizations clearly show the effect of forcing on flow structures and the strong correlation of number of coherent structures and Rossiter modes, even when the modes are not dominant.

\section{Acknowledgments}

This work was supported in part by the AFRL/VA and AFOSR through the Collaborative Center of Control Science (Contract F33615-01-2-3154), and by DAGSI. The simulation work is supported in part under NASA's Quiet Aircraft Technology Program and in part by a grant of computer time from the DOD High Performance Computing Modernization Program at the Army Research Laboratory. The help of Jeff Kastner, Jim Hileman, and Brian Thurow, graduate students at
GDTL, with the experimental aspects of the work is greatly appreciated.

\section{References}

Adrian, R. J., "On the Role of Conditional Averages in Turbulent Theory," Turbulence in Liquids, Science Press, Princeton, 1977.

Adrian, R.J. and Moin, P., "Stochastic estimation of organized turbulent structure: Homogeneous shear Flow," Journal of Fluid Mechanics, Vol. 190, 1988, pp. 531-559.

Baldwin, B. S., and Lomax, H., "Thin-Layer Approximation and Algebraic Model for Separated Turbulent Flows," AIAA Paper 78-257, January 1978 .

Caraballo, E., Samimy, M., and DeBonis, J., "Low Dimensional Modeling of Flow for Closed-Loop Flow Control," AIAA Paper 2003-0059, January 2003.

Cattafesta, L. N., III, Garg, S., Choudhari, M., and Li, F., "Active Control of Flow-Induced Cavity Response,” AIAA Paper 97-1804, June-July 1997.

Cattafesta, L. N., III, Garg, S., Kegerise, M. A., and Jones, G. S., "Experiments on Compressible FlowInduced Cavity Oscillations," AIAA Paper 98-2912, June 1998.

Cattafesta, L. N., III, Williams, D. R., Rowley, C. W., and Alvi, F. S., "Review of Active Control of FlowInduced Cavity Resonance," AIAA Paper 2003-3567, June 2003.

Cole, D.R. and Glauser, M.N, "Application of Stochastic Estimation in the axisymmetric Sudden Expansion," Physics of Fluids, Vol. 10, No. 11, 1998, pp. 2941-2949.

Cole, D.R., Glauser, M.N. and Guezennec, Y.G., "An Application of the Stochastic Estimation to the Jet Mixing Layer," Physics of Fluids, Vol. 4, No. 1, 1991, pp. 192-194.

Debiasi, M. and Samimy, M., "An Experimental Study of the Cavity Flow for Close-Loop Flow Control," AIAA Paper 2003-4003, June 2003.

DeBonis, J.R. and Scott, J.N., "A Study of the Error and Efficiency of Numerical Schemes for Computational Aeroacoustics," AIAA Journal, Vol. 40, No. 2, pp. 227-234, 2002a.

DeBonis, J.R. and Scott, J.N., "Large-Eddy Simulation of a Turbulent Compressible Round Jet," AIAA Journal, Vol. 40, No. 7, pp. 1346-1354, 2002 b.

Efe, M.Ö. and Özbay, H., "Proper Orthogonal Decomposition for Reduced Order Modeling: 2D Heat Flow," to appear in the Proc. of IEEE Int. Conf. on Control Applications (CCA'2003), June 23-25, Istanbul, Turkey, 2003a.

Efe, M.Ö. and Özbay, H., "Integral Action Based Dirichlet Boundary Control of Burgers' Equation," to 
appear in the Proc. of IEEE Int. Conf. on Control Applications (CCA'2003), June 23-25, Istanbul, Turkey, 2003b.

Efe, M. Ö, X. Yuan, H. Özbay, and M. Samimy, "Interpolating the Local Models of POD Using Fuzzy Decision Mechanisms," submitted to $9^{\text {th }}$ Mechatronics Forum, Ankara, Turkey, August-September 2004a.

Efe, M. Ö., M. Debiasi, H. Özbay, and M. Samimy, "Modeling of Subsonic Cavity Flows by Neural Networks," submitted to Int. Conf. on Mechatronics (ICM'04), İstanbul, Turkey, June 2004b.

Kennedy, C.A. and Carpenter, M.H., "Comparison of Several Numerical Methods for Simulation of Compressible Shear Layers," NASA TP 3483, December 1997.

Murray, N. and Ukeiley, L, "Estimation of the Flowfield from Surface Pressure Measurements in an Open Cavity," AIAA Journal, Vol. 41, No. 5: technical notes, May 2003, pp. 969-972.

Narendra, K.S. and Parthasarathy, K., "Identification and Control of Dynamical Systems Using Neural Networks," IEEE Trans. on Neural Networks, Vol. 1, No. 1, 1990, pp. 4-27.

Patel, M. P., Kolacinski, R. M., Prince, T. S., Ng, T. T., and Cain, A. B., "Flow Control Using Intelligent Control Modules for Virtual Aerodynamic Shaping," AIAA Paper 2003-3663, June 2003.

Picard, C. and Delville, J., "Pressure Velocity Coupling in a Subsonic Round Jet;" International Journal of Heat and Fluid Flow, Vol. 21, pp. 359-364, 2000.

Rowley, C. W., Colonius, T., and Murray, R. M., "Dynamical Models for Control of Cavity Oscillations," AIAA Paper 2001-2126, May 2001.

Rowley, C. W., Williams, D. R., Colonius, T., Murray, R. M., MacMartin, D. G., and Fabris, D., "ModelBased Control of Cavity Oscillations Part II: System Identification and Analysis," AIAA Paper 2002-0972, January 2002a.

Rowley, C. W., Colonius, T. and Basu, A., "On SelfSustained Oscillations in Two-Dimensional
Compressible Flow Over Rectangular Cavities," Journal of Fluid Mechanics, Vol. 455, 2002b, pp. 315-346.

Rowley, C. W. and Williams, D. R, "Control of Forced and Self-Sustained Oscillations in the Flow Past a Cavity," AIAA Paper 2003-0008, January 2003.

Samimy, M., Debiasi, M., Caraballo, E., Özbay, H., Efe, M. Ö., Yuan, X., DeBonis, J., and Myatt, J.H., "Closed-Loop Active Flow Control - A Collaborative Approach," AIAA Paper 2003-0058, January 2003a.

Samimy, M., Debiasi, M., Caraballo, E., Özbay, H., Efe, M. Ö., Yuan, X., DeBonis, J., and Myatt, J.H., "Development of Closed-loop Control for Cavity Flows," AIAA Paper 2003-4258, June 2003b.

Sirovich, L., "Turbulence and the Dynamics of Coherent Structures," Quarterly of Applied Mathematics, Vol. XLV, No. 3, 1987, pp. 561-590.

Traub, L. W., Miller, A., Ukpai, U. I., Rediniotis, O., Jeong, G., and Kim, K., "Reconfigurable Synthetic Jet Actuation and Closed-Loop Flow Control," AIAA Paper 2003-0217, January 2003.

Williams, D. R., Fabris, D., and Morrow, J., "Experiments on Controlling Multiple Acoustic Modes in Cavities," AIAA Paper 2000-1903, June 2000.

Williamson, J.H., "Low-Storage Runge-Kutta Schemes," Journal of Computational Physics, vol. 35, pp 48-56, 1980.

Yan, P., M. Debiasi, X. Yuan, E. Caraballo, M.Ö. Efe, H. Özbay, M. Samimy, J. DeBonis, C. Camphouse, J.H. Myatt, A. Serrani and J. Malone, "Controller Design for Active Closed-Loop Control of Cavity Flows," AIAA Paper 2004-0573, January 2004.

Yuan, X., Efe, M.Ö., and Özbay, H., "On Delay-Based Linear Models and Robust Control of Cavity Flows," presented at the NSF-CNRS Workshop on Advances in Time Delay Systems, France, Jan. 22-24, 2003. 

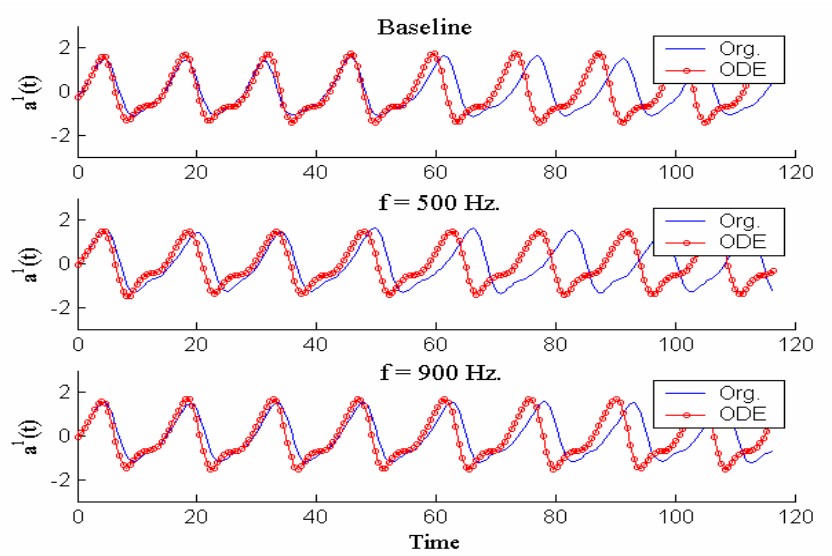

Figure 3.1 Evolution of the first time coefficient for the model obtained solving Eqn. 3.5 with the explicit control input using 5 POD modes.

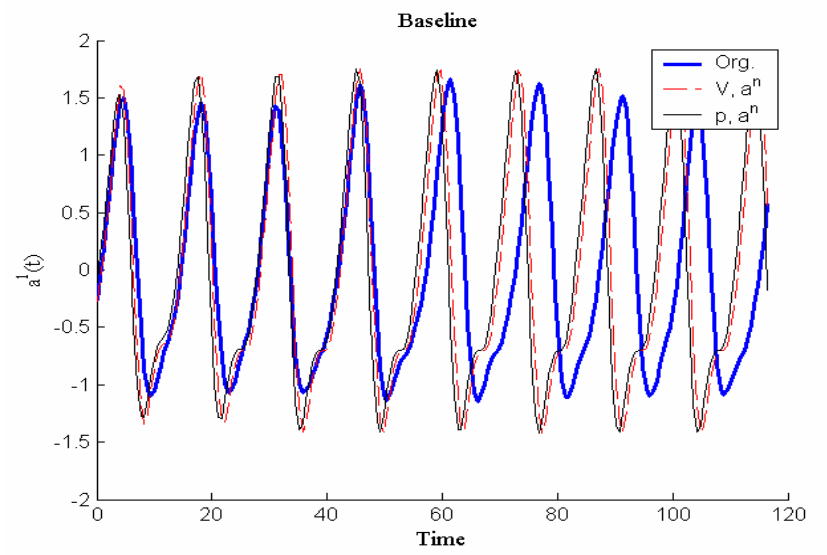

Figure 3.2 Evolution of the first time coefficient for the baseline case using 5 POD modes by: projecting the flow field onto the POD basis (thick line); using Eqns. 3.4 with initial conditions from flow field (dashed line); using as initial conditions the flow filed estimated from pressure measurements (thin line).
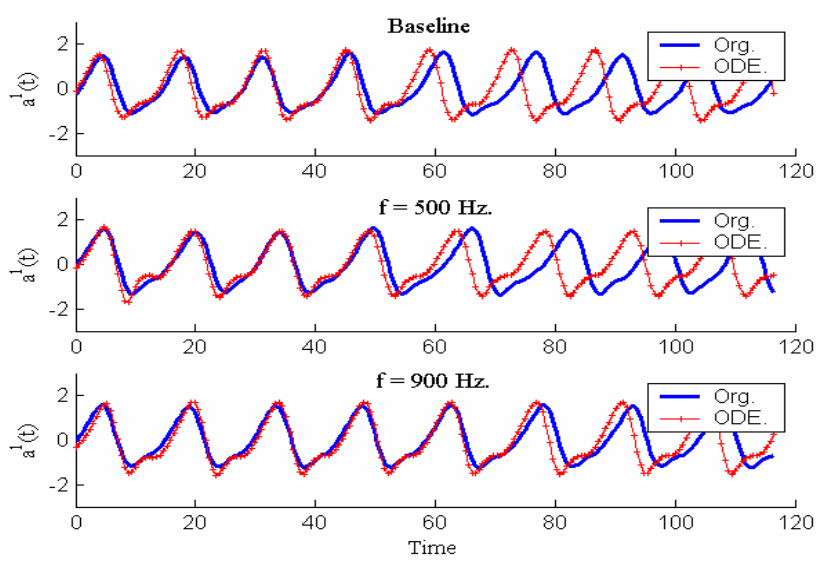

Figure 3.3 Evolution of the first time coefficient for the baseline and forced cases using 5 POD modes by projecting the flow field onto the POD basis (thick line) and by using as initial conditions the flow field estimated from pressure measurements (thin line).

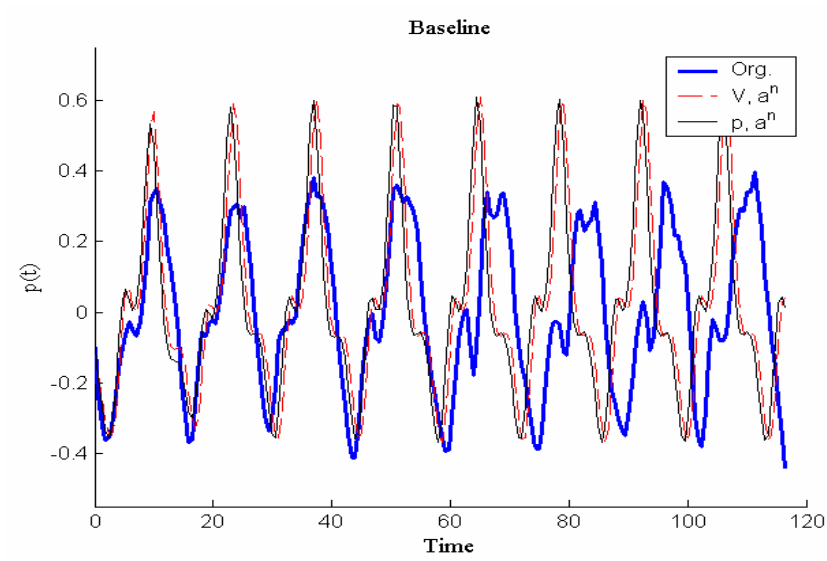

Figure 3.4 Evolution of the cavity floor pressure fluctuations estimated for the baseline case by using 5 POD modes.
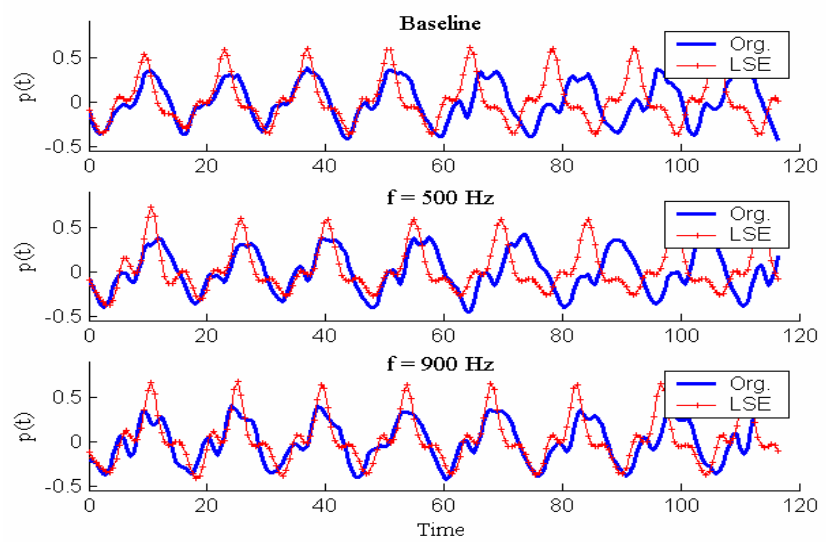

Figure 3.5 Evolution of the cavity floor pressure fluctuations estimated for the baseline and forced cases by using 5 POD modes. 


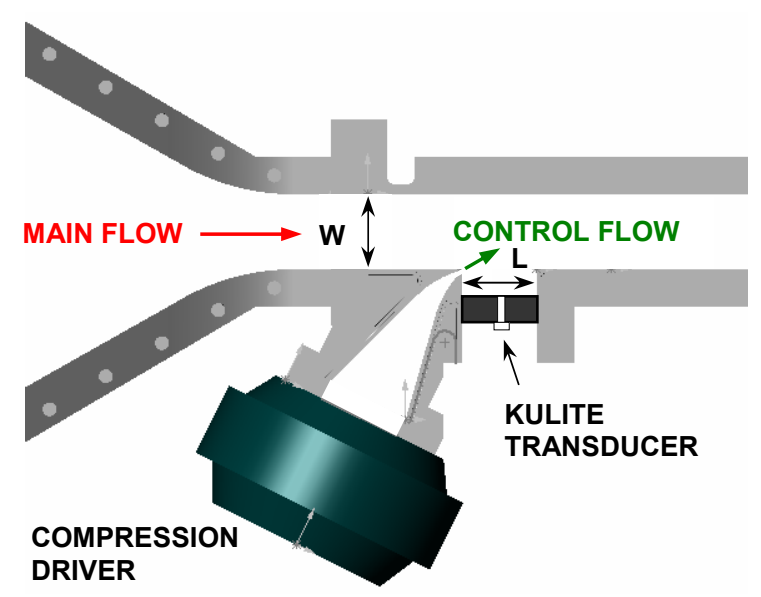

Figure 5.1: Cutout of the facility showing the converging nozzle, the test section, the cavity, the actuator layout, and the placement of the Kulite transducer.

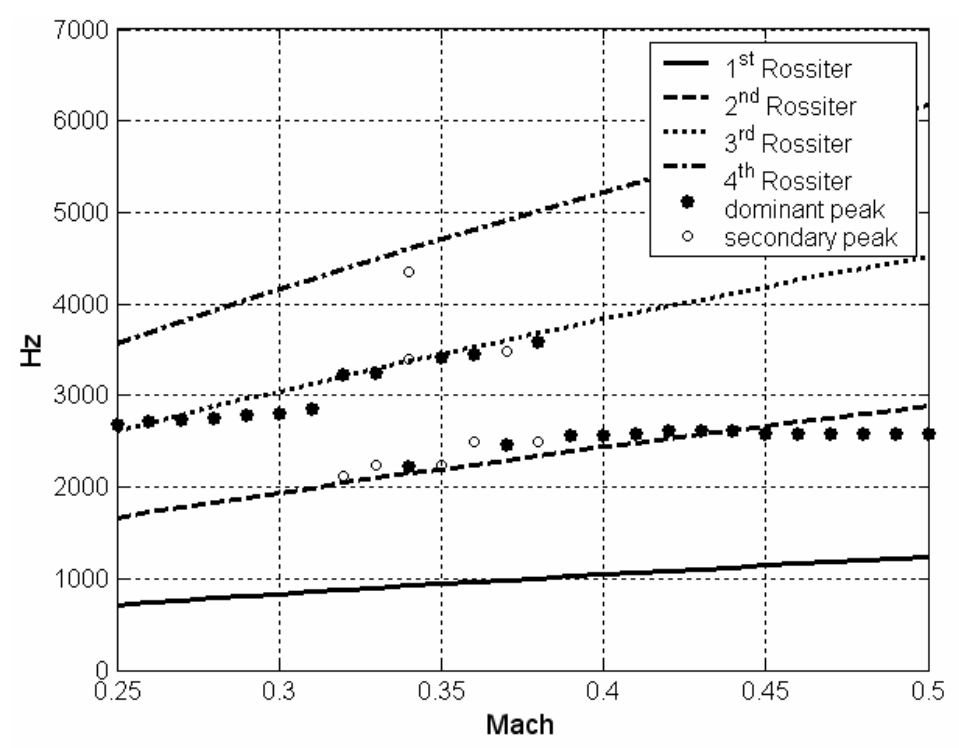

resonant frequencies (circles) as a function of the flow Mach number.

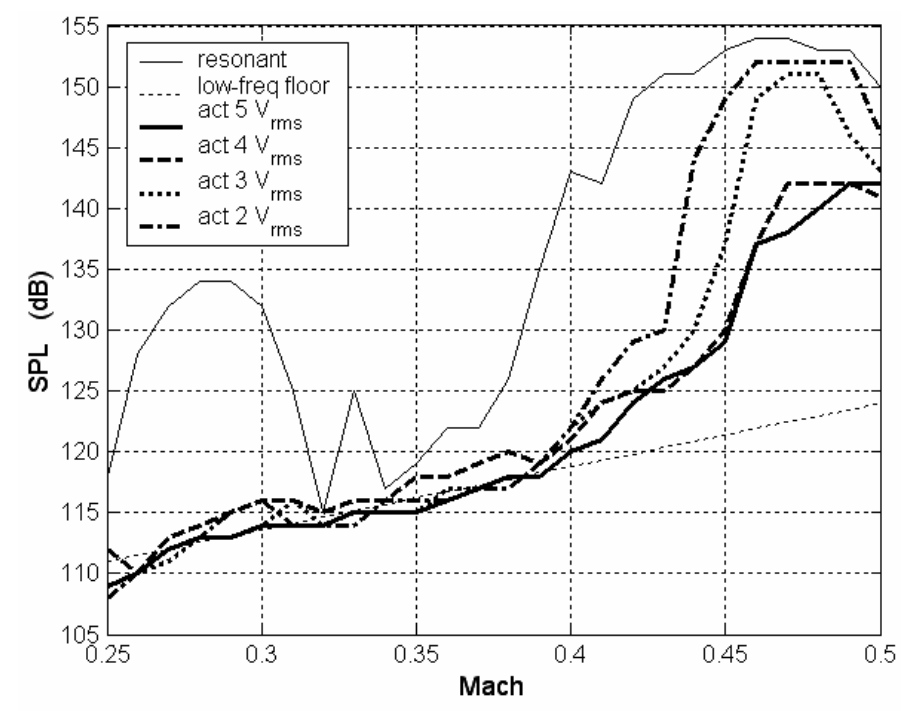

Figure 5.3: Amplitude of the dominant pressure peak at the cavity floor for unforced flow and of the flow forced at optimal frequency for peak reduction as a function of the flow Mach number.

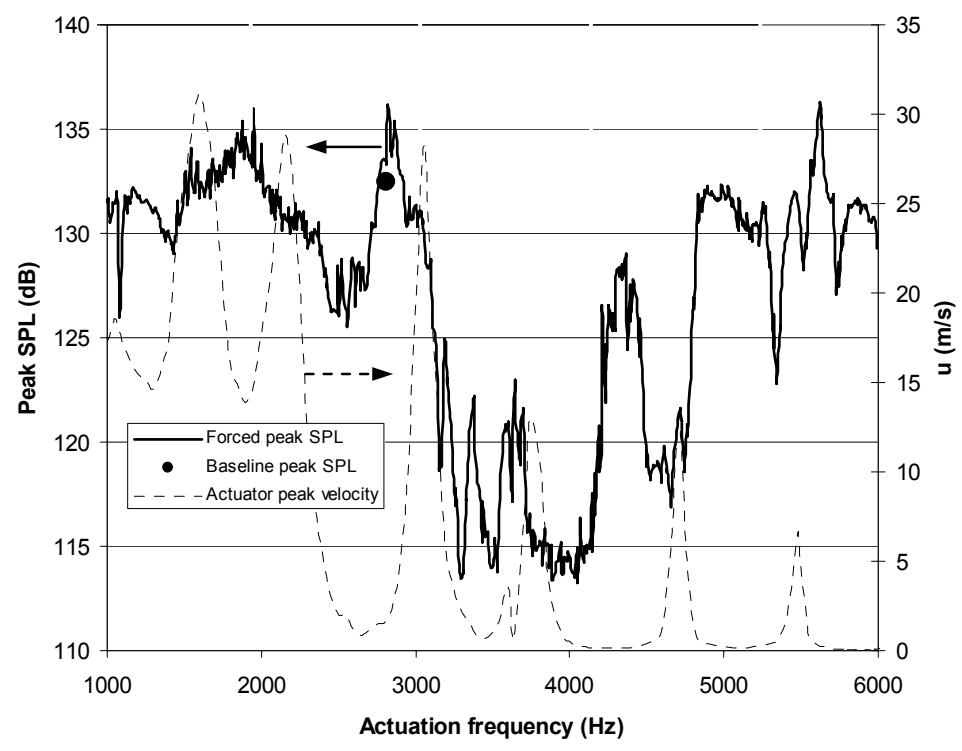

flow with frequency of sinusoidal forcing at $4 \mathrm{~V}_{\text {rms }}$. Continuous thick line is the peak SPL at the cavity floor and dotted thin line is the exit-slot velocity for actuation at 4 $\mathrm{V}_{\text {rms. }}$. 

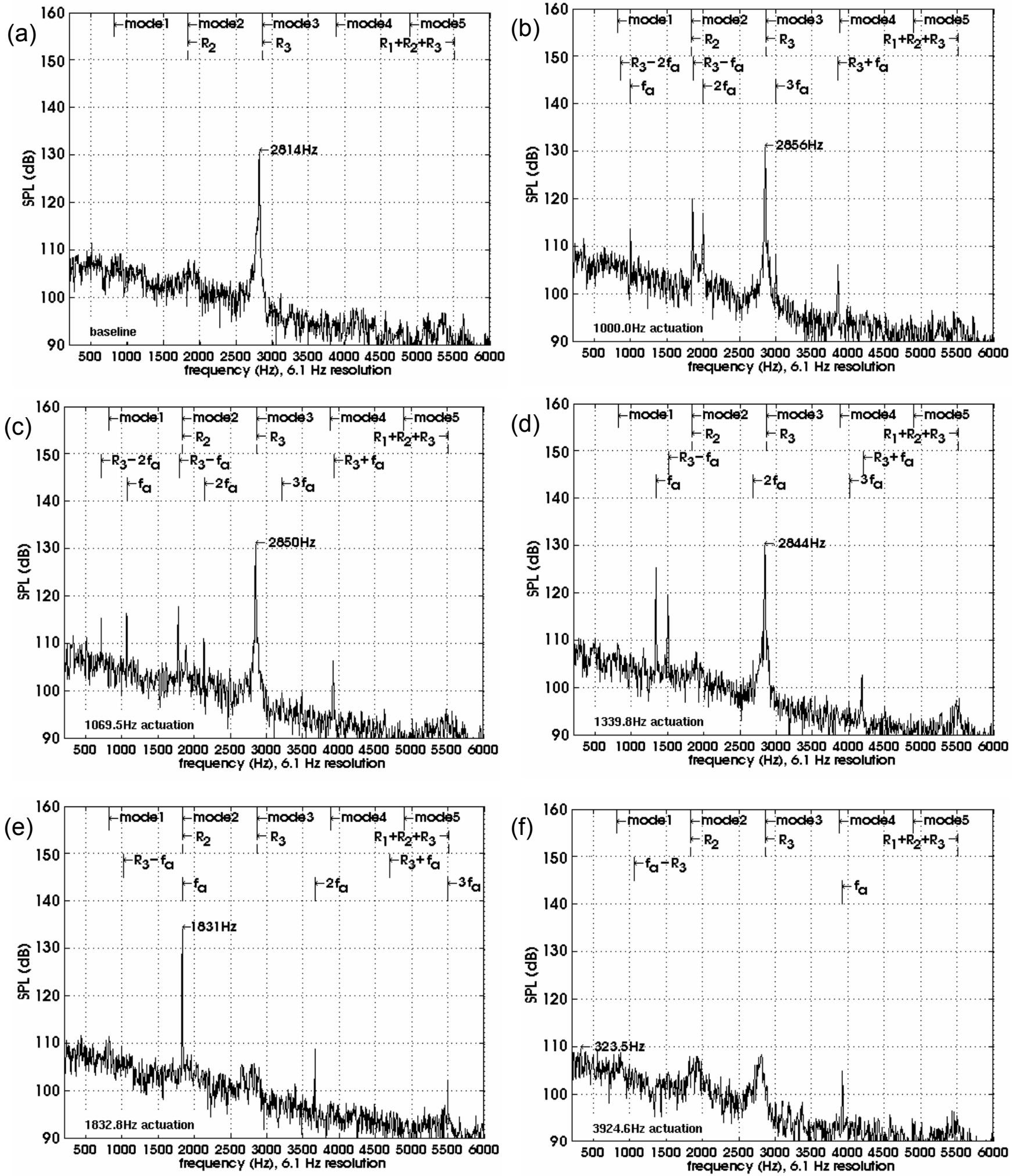

Figure 5.5: Cavity floor pressure spectra of the Mach 0.3 cavity flow under various forcing conditions at $4 \mathrm{~V}_{\text {rms: }}$ : (a) no forcing (baseline); (b) $1000 \mathrm{~Hz}$; (c) $1070 \mathrm{~Hz}$; (d) $1340 \mathrm{~Hz}$; (e) $1830 \mathrm{~Hz}$; (f) $3920 \mathrm{~Hz}$ (optimal forcing frequency). $R_{1}, R_{2}, R_{3}$ indicate the Rossiter frequencies, $f_{a}$ the actuation frequency. 

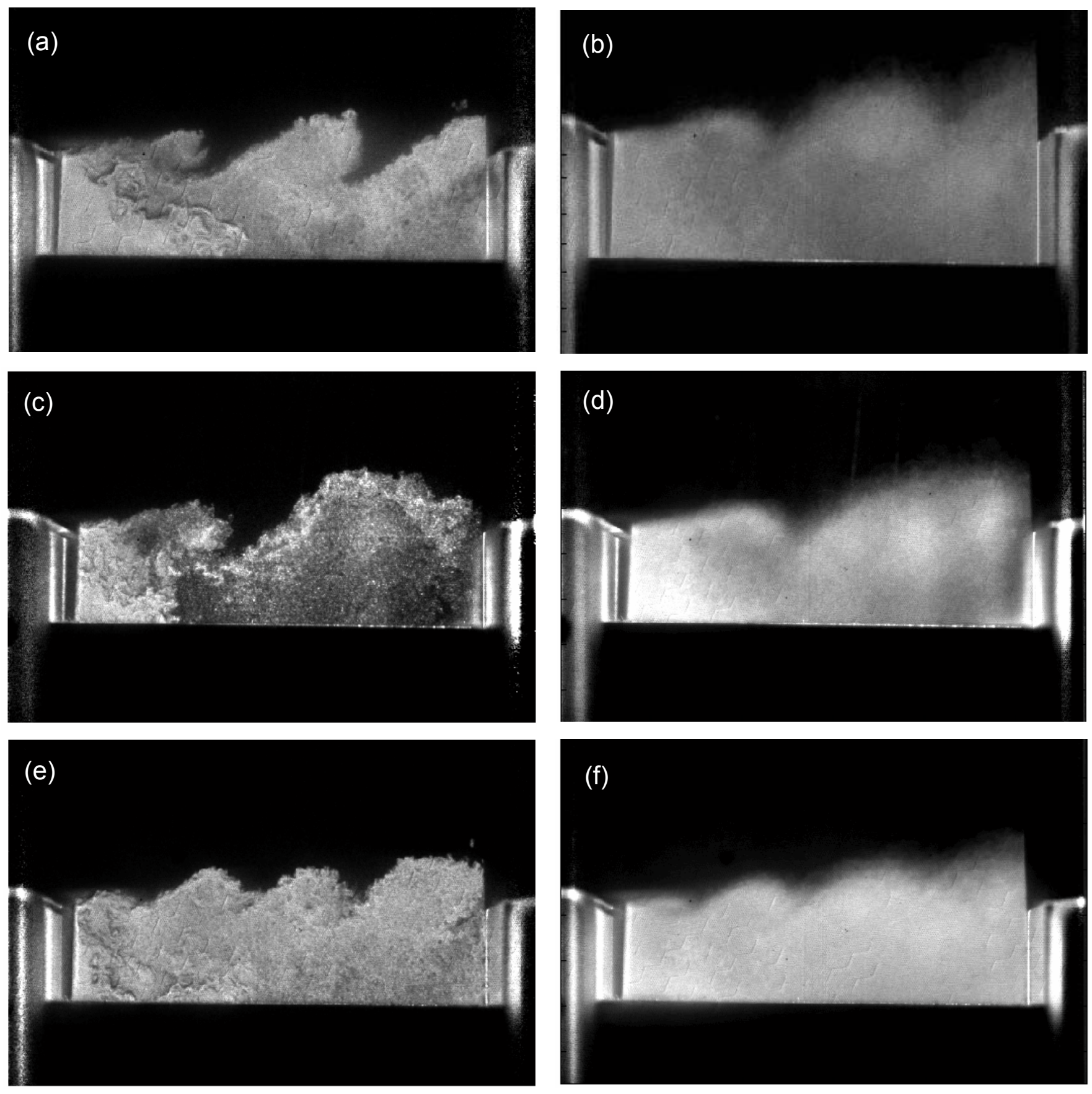

Figure 5.6: Instantaneous (left) and phase-locked average (right) laser light scattering images of the Mach 0.3 cavity flow under various forcing conditions: (a) and (b) no forcing (baseline); (c) and (d) $1830 \mathrm{~Hz}$ forcing ( ${ }^{\text {nd }}$ Rossiter mode) at $4 \mathrm{~V}_{\mathrm{rms}}$; (e) and (f) $3920 \mathrm{~Hz}$ forcing at $4 \mathrm{~V}_{\mathrm{rms}}\left(4^{\text {th }}\right.$ Rossiter mode and optimal forcing frequency). Flow is from left to right. 

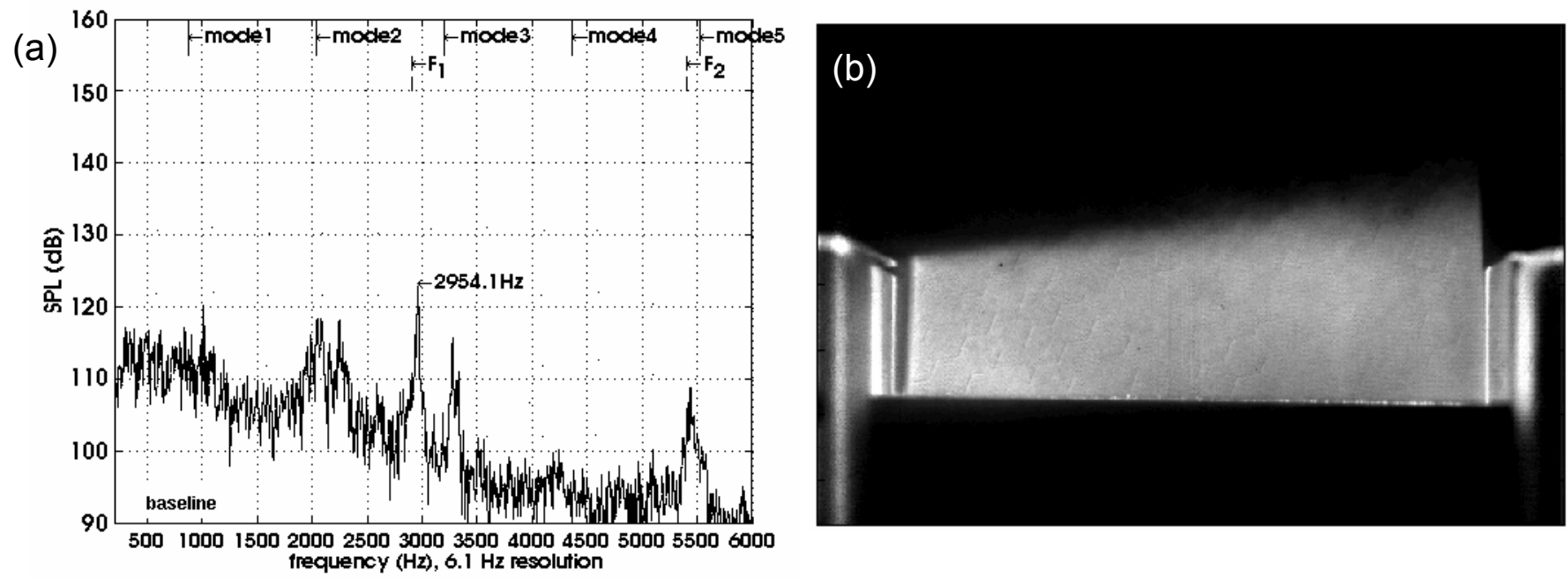

Figure 5.7: Cavity floor pressure spectra of the unforced Mach 0.32 cavity flow (left) and average laser light scattering image (right) of the same flow. $\mathrm{F}_{1}$ and $\mathrm{F}_{2}$ indicate frequencies not associated to resonance. Flow is from left to right.
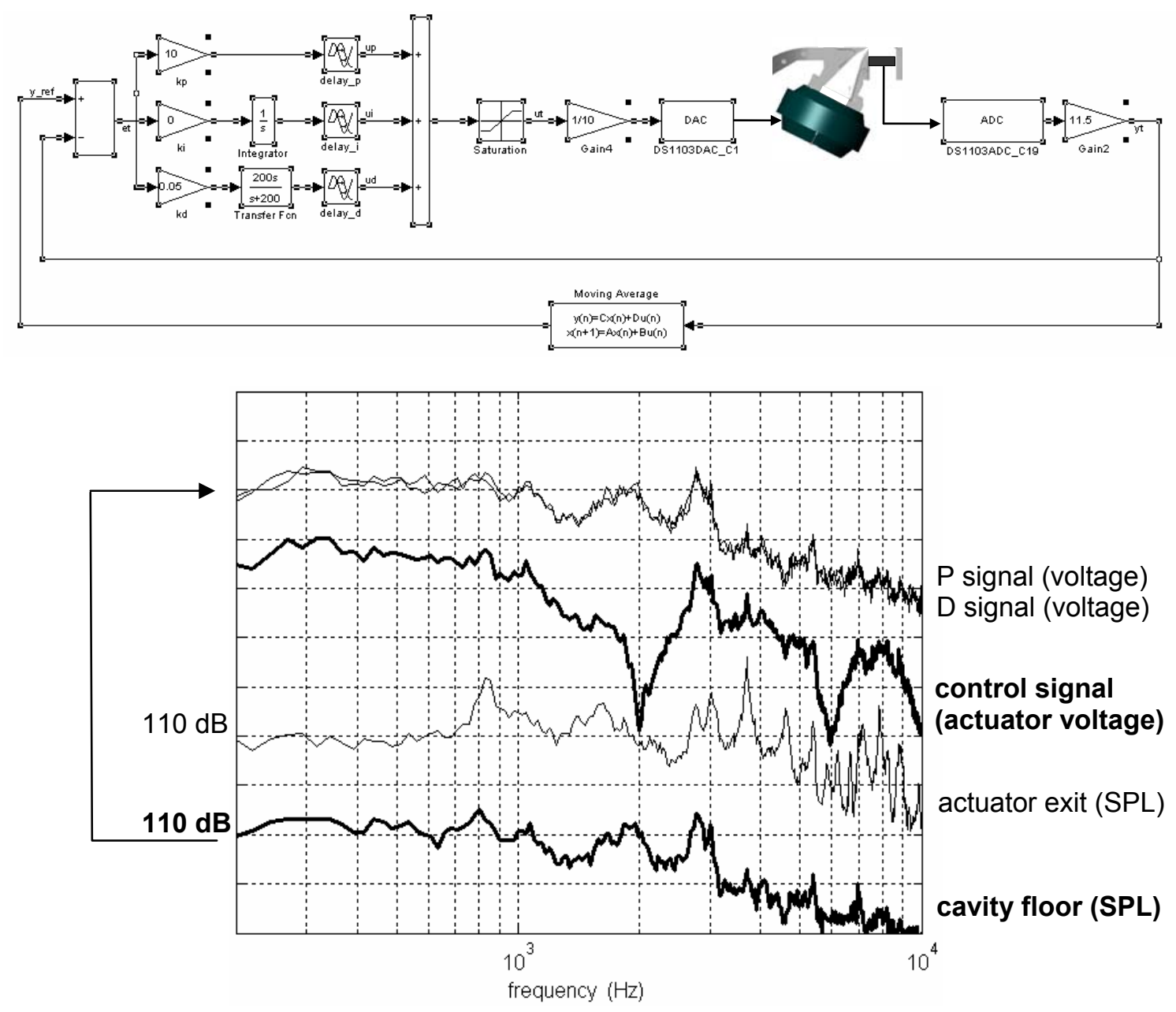

Figure 5.8: Spectra for Mach 0.30 cavity flow system excited by the PD like controller with time delay of $260 \mu \mathrm{s}$ between the $\mathrm{P}$ and $\mathrm{D}$ terms. The $\mathrm{P}, \mathrm{D}$, and control signals were processed as the pressure transducers signals. Reference dB levels are provided only for the SPL spectra of the pressure transducer signals. 